\title{
Belatedly diagnosed acute motor axonal neuropathy after cardiac surgery
}

\author{
Chong Tae Kim ${ }^{1,2}$, Todd Beery ${ }^{2}$ \\ ${ }^{1}$ Division of Rehabilitation Medicine, The Children's Hospital of Philadelphia, Philadelphia, USA; \\ *Corresponding Author: kim@email.chop.edu \\ ${ }^{2}$ Department of Physical Medicine and Rehabilitation, University of Pennsylvania, Philadelphia, USA
}

Received 18 March 2013; revised 18 April 2013; accepted 10 May 2013

Copyright @ 2013 Chong Tae Kim, Todd Beery. This is an open access article distributed under the Creative Commons Attribution License, which permits unrestricted use, distribution, and reproduction in any medium, provided the original work is properly cited.

\begin{abstract}
Surgery has been reported a rare cause of Guillain-Barré syndrome (GBS), but a recent retrospective study reported a much higher incidence rate for post-surgical patients. There are several case reports of GBS presenting after cardiac surgical procedures. All these cases were diagnosed as acute inflammatory demyelinating polyradiculoneuropathy (AIDP). We described a case of acute motor axonal neuropathy (AMAN) after cardiac surgery. Clinical features were reviewed along with spinal magnetic resonance imaging (MRI) and cerebral spinal fluid (CSF) analysis. Sequential electrodiagnostic studies (EDx) were performed. This case represented a rare complication of AMAN with urinary retention after cardiac surgery.
\end{abstract}

Keywords: Guillain-Barré Syndrome; Acute Motor Axonal Neuropathy; Cardiac Surgery; Sequential Electrodiagnosis; Urinary Retention

\section{INTRODUCTION}

Gullain-Barre syndrome (GBS) most commonly develops following antecedent upper respiratory or gastrointestinal infections. Other risk factors for GBS include vaccination and surgery. GBS is classified into three types, based on electrodiagnostic findings, which inclued acute inflammatory demyelinating polyradiculoneuropathy (AIDP), acute motor axonal neuropathy (AMAN), and acute motor sensory axonal neuropathy (AMSAN). AIDP is the most common, and AMSAN is the rarest. Several cases of post-operative GBS have been reported [1-6]. To our knowledge, there are no reported cases of AMAN after an operative procedure. Herein, we reported a case of AMAN with urinary re- tention that developed after cardiac surgery. The diagnosis of AMAN was confirmed by serial electrodiagnostic studies (EDx).

\section{CASE PRESENTATION}

A 24-year-old female with congenital heart disease (tetralogy of Fallot and absent pulmonic valve) and a history of prior cardiac heart surgery (main pulmonary artery banding, ventricular septal defect closure, right ventricle to pulmonary artery conduit insertion, and right pulmonary artery placation) as a 2-year-old presented to a cardiology department of a university children's hospital with a gradual decline in general strength and endurance. Prior to developing these symptoms, her physical function was normal. She was found to have interval changes of pulmonary stenosis, tricuspid regurgitation, and aneurismal formation of the left pulmonary artery. Consequently, she underwent an elective pulmonic valve replacement, revision of the right ventricle to pulmonary artery conduit, and left pulmonary artery placation under general anesthesia. The procedure was completed using the standard cardiopulmonary bypass and was uneventful. Preoperatively, she denied having any respiratory or gastrointestinal symptoms. Cefazolin was given prior to the procedure. Total bypass time of the procedure was 89 minutes, and no blood products were transfused. She was extubated without complications. The medications given postoperatively consisted of intravenous vasopressors (discontinued within 24 hours), furosemide, docusate, senna, nalbuphine hydrochloride, and hydromorphone. On postoperative day 2, she complained of paresthesia and weakness in her bilateral lower extremities. Later that day, she was unable to stand without assistance and had bilateral foot drop. On physical exam, manual muscle testing revealed 2/5 muscle strength in ankle dorsiflexors and 4/5 elsewhere in the lower extremities. Both ankle and knee reflexes were not elicited. Cranial nerves remained intact 
and upper extremities were full strength. Sensations to pain, light touch, proprioception, and vibration were all preserved symmetrically. An initial magnetic resonance imaging (MRI) of the thoracolumbar spine performed on post-operative day 3 indicated no evidence of spinal cord infarction or abnormal contrast enhancement involving the cauda equina. Her post-operative course was also significant for urinary retention, and she was treated for a urinary tract infection with oral antibiotics (cotrimoxazole $160 \mathrm{mg}$ twice a day) for ten days, however urinary retention continued to persist. Over the next several days, lower extremity weakness progressed to $4 / 5$ in hip flexors, 3/5 in knee extensors, and 0/5 in ankle dorsiflexors, and 2/5 ankle plantar flexors. On post-operative day 5, an initial EDx was performed and motor nerves (bilateral peroneal, right tibial, and right ulnar nerves), sensory nerves (right radial and sural nerves) conduction studies, and $\mathrm{F}$ waves (right tibial and ulnar nerves) were normal, respectively. A repeat thoracolumbar MRI on post-operative day 6 demonstrated disseminated enhancement of the peripheral nerve roots of the cauda equina. Lumbar puncture was performed on post-operative day 7 and CSF analysis showed elevated protein (86 mg/dL), normal white cell count, normal glucose (55 mg/dL), negative oligoclonal bands, and elevated myelin basic protein $(8.10 \mathrm{ng} / \mathrm{mL})$. Compylobacter testing was not performed. For treatment, a 5-day course of intravenous immunoglobulin (400 mg/kg/day) was completed with no clinical improvement. 13 days after her cardiac procedure, she was admitted to an inpatient rehabilitation unit with continued bilateral lower extremity weakness and urinary retention.

During inpatient rehabilitation, she did not make sig- nificant improvements, and her bilateral lower extremity weakness and urinary retention persisted. A second EDx was performed 7 weeks post-operatively showed remarkably decreased amplitudes of compound muscle action potentials (CMAP) of the bilateral peroneal nerves. Right tibial CMAP amplitude was slightly decreased, and conduction velocities of the bilateral tibial nerves were minimally slowed. F wave latency of the left tibial nerve by stimulation at the ankle was within normal ranges (Table 1). No conduction blocks or temporal dispersions were observed. Sensory nerve action potentials (SNAP) were within normal ranges in the bilateral sural and left radial, median, and ulnar nerves. Copious fibrillation potentials and positive sharp waves with neurogenic recruitment patterns were noted in the left lower extremity (tibialis anterior, gastrocnemius, biceps femoris, and vastus medialis) and less in the lumbar paraspinal muscles. These findings were consistent with AMAN. At the time of discharge from inpatient rehabilitation, she continued to have bilateral lower extremity weakness and paresthesia in a similar pattern as onset. She used loftstrand crutches to assist with ambulation, and also continued to suffer from a neurogenic bladder, requiring intermittent self-catheterization.

\section{DISCUSSION}

Post-operative acute peripheral neuropathy may develop after any surgical procedure. It can be caused by various injury mechanisms, which includes mechanical injury, ischemia, and altered immune system function that causes an inflammatory neuropathy. The last is believed to be the pathophysiology of GBS after surgery.

Table 1. Electrodiagnostic study.

\begin{tabular}{|c|c|c|c|c|}
\hline Side & Nerve $(\mathrm{M} / \mathrm{S})^{*}$ & Distal latency (R/Ref) ${ }^{\dagger}$ (msec) & Distal amplitudes (R/Ref) & Conduction velocity (R/Ref) (m/s) \\
\hline \multirow[t]{2}{*}{ Right } & Tibial (M) & $5.9 /<6.1$ & $2.7 / 3 \mathrm{mV}$ & $38 />40$ \\
\hline & Peroneal (M) & $5.3 /<6.5$ & $0.55 / 2 \mathrm{mV}$ & $46.2 />40$ \\
\hline \multirow[t]{4}{*}{ Left } & Tibial (M) & $5.1 /<6.1$ & $3.8 / 3 \mathrm{mV}$ & $39.5 />40$ \\
\hline & Peroneal (S) & $5.4 /<6.5$ & $0.69 / 2 \mathrm{mV}$ & $42 />40$ \\
\hline & Ulnar(M) & $3.3 /<3.5$ & $8 / 6 \mathrm{mV}$ & $58.2 />50$ \\
\hline & Median(M) & $4.4 /<4.2$ & $4.3 / 4 \mathrm{mV}$ & $60 />50$ \\
\hline Right & Sural (S) & $4.3 /$ & $35.9 \mu \mathrm{V}$ & \\
\hline \multirow[t]{3}{*}{ Left } & Sural (S) & $4.3 /$ & $27.7 \mu \mathrm{V}$ & \\
\hline & Ulnar (S) & $3.5 /$ & $18.7 \mu \mathrm{V}$ & \\
\hline & Median (S) & $3.5 /$ & $31.7 \mu \mathrm{V}$ & \\
\hline Left & Tibial $^{\ddagger}$ & $49.5 / 42-56$ & & \\
\hline
\end{tabular}

\footnotetext{
"M/S, motor/sensory; ${ }^{\dagger} \mathrm{R} /$ Ref, recorded/reference value; ${ }^{\ddagger}$, F wave.
} 
Cases of GBS developing after surgery are reported with incidence of $5 \%-10 \%$, and are not related to the type of anesthesia or surgical procedure [1,2]. A recent retrospective study revealed the relative risk of developing GBS within the post-operative 6-week period is 13.1 times higher than the general population incidence rate [2]. All previous cases of GBS after surgery were reported as AIDP with one exception. A case of AMSAN was reported after a thoracic spine surgery [7]. AMAN is known to progress faster and reach nadir earlier than AIDP [8]. This holds true when comparing the onset times of the cases of post-operative AIDP to the onsets of our case and the one case of AMSAN [7] (AIDP postoperative onset 6 - 40 days versus AMAN and AMSAN 6 - 48 hours). More substantial data is needed to determine that AMAN or AMSAN develop earlier than AIDP after surgery.

The pathophysiology of post-operative GBS is not entirely clear. Histologic studies reveal infiltration of macrophages, causing inflammation into the nerve [1]. The location of the infiltration being in either the axon or myelin can assist in distinguishing the various subtypes. Macrophages invade the space between the Schwann cell and axon not involving myelin in AMAN [9]. Two immune response hypotheses have been proposed to explain the infiltration process. One of these involves a T-cell response against antigens on the nerve surface causing a release of inflammatory mediators [10]. Another theory is based on a humoral response from antibodies binding to epitopes on the nerve surface, causing activation of the complement cascade [11]. There is no clear understanding on how the two entities are related. The existing theories regarding the pathophysiology revolve around the principle that surgery inherently results in systemic inflammation [12-14]. The causes of inflammation can possibly be induced by ischemia, reperfusion injury, and the general trauma of the surgery, which can consequently cause a humoral and cytokine response by the immune system. The inflammatory response after a cardiopulmonary surgery has been shown to activate the complement system, causing an increase of cytotoxic mediators and activation of macrophages, neutrophils, and T-cells [15]. However, there is no clear evidence indicating that activating these cells is directly related to the occurrence of GBS following cardiopulmonary bypass surgery.

If axonal lesions are limited to the distal segments, the recovery is favorable because of good capability of axonal regeneration [16-18]. The poor recovery of our case can be explained by the lesions involved in proximal nerve roots as well as distal segments of the peripheral nerves. As demonstrated in this case, sequential EDx is necessary to confirm GBS and to classify subtypes, especially since $10 \%$ of cases of GBS have a normal EDx in the early phase $[19,20]$.

Urinary dysfunction is a common impairment associated with GBS. The incidence of the impairment is reported in 20\% of AIDP cases and 50\% of AMAN cases [21]. Urodynamic studies have demonstrated both detrusor overactive and underactive bladders in cases of GBS [21-23]. Cases of detrusor overactivity in peripheral nerve lesion is paradoxical, but have been reported in cauda equina and pelvic plexus lesions [24,25]. Possible explanations for detrusor overactivity include pelvic nerve irritation, emphatic transmissions, or an undefined mechanism [21]. In our case, since the patient deferred urodynamic study, it is unclear whether the bladder was overactive or underactive. Since the patient had sustained urinary retention, it is assumed to be an underactive bladder.

\section{CONCLUSION}

To our knowledge, five cases of AIDP were reported after cardiac operative procedures. This is the first report of AMAN after a cardiac operative procedure, and required sequential EDx for diagnosis.

\section{REFERENCES}

[1] Arnason, B.G. and Asbury, A.K. (1968) Idiopathic polyneuritis after surgery. Archives of Neurology, 18, 500-507. http://dx.doi.org/10.1001/archneur.1968.00470350058005

[2] Gensicke, H., Datta, A.N., Dill, P., Schindler, C. and Fischer, D. (2012) Increased incidence of Guillain-Barre syndrome after surgery. European Journal of Neurology, 19, 1239-1244.

http://dx.doi.org/10.1111/j.1468-1331.2012.03730.x

[3] Renlund, D.G., Hanley, D.F. and Traill, T.A. (1987) Guillain-Barre syndrome following coronary artery bypass surgery. American Heart Journal, 113, 844-845. http://dx.doi.org/10.1016/0002-8703(87)90736-8

[4] Campbell, A.J., McKinlay, K. and Scott, N.B. (2009) Guillain-Barre syndrome after cardiopulmonary bypass. Journal of Cardiothorac Vascular Anesthesia, 23, 82-83. http://dx.doi.org/10.1053/j.jvca.2008.04.019

[5] Hogan, J.C., Briggs, T.P. and Oldershaw, P.J. (1992) Guillain-Barre syndrome following cardiopulmonary bypass. International Journal of Cardiology, 35, 427-428. http://dx.doi.org/10.1016/0167-5273(92)90248-2

[6] Costantino, T. and Weintraub, A. (1972) The GuillainBarre syndrome as a complication of the postperfusion syndrome. American Heart Journal, 84, 678-680. http://dx.doi.org/10.1016/0002-8703(72)90183-4

[7] Cheng, J., Kahn, D.E. and Wang, M.Y. (2011) The acute motor-sensory axonal neuropathy variant of GuillainBarre syndrome after thoracic spine surgery. Journal of Neurosurgery and Spine, 15, 605-609. http://dx.doi.org/10.3171/2011.8.SPINE1159

[8] Hiraga, A., Mori, M., Ogawara, K., Hattori, T. and Ku- 
wabara, S. (2003) Differences in patterns of progression in demyelinating and axonal Guillain-Barre syndrome. Neurology, 61, 471-474. http://dx.doi.org/10.1212/01.WNL.0000081231.08914.A $\underline{1}$

[9] Griffin, J.W., Li, C.Y., Macko, C., Ho, T.W., Hsieh, S.T., Xue, P., et al. (1996) Early nodal changes in the acute motor axonal neuropathy pattern of the Guillain-Barre syndrome. Journal of Neuroctyology, 25, 33-51. http://dx.doi.org/10.1007/BF02284784

[10] Archelos, J.J., Previtali, S.C. and Hartrung, H.-P. (1999) The role of integrins in immune-mediated diseases of the nervous system. Trends in Neuroscience, 22, 30-38. http://dx.doi.org/10.1016/S0166-2236(98)01287-9

[11] Yuki, N. (2001) Infectious origins of, and molecular mimicry in, Gullain-Barre and Fisher syndromes. Lancet Infectious Disease, 1, 29-37. http://dx.doi.org/10.1016/S1473-3099(01)00019-6

[12] Hartung, H.P., Willison, H.J. and Kieseier, B.C. (2002) Acute inflammatory neuropathy: Update on GuillainBarre syndrome. Current Opinions in Neurology, 15, 571577. http://dx.doi.org/10.1097/00019052-200210000-00008

[13] Asimakopoulos, G. (2001) Systemic inflammation and cardiac surgery. Perfusion, 16, 353-360. http://dx.doi.org/10.1177/026765910101600505

[14] Staff, N.P., Engelstad, J.N., Klein, C.J., Amrami, K.K., Spinner, R.J., Dyck, P.J., et al. (2010) Post-surgical inflammatory neuropathy. Brain, 133, 2866-2880. http://dx.doi.org/10.1093/brain/awq252

[15] Hogan, B.V., Peter, M.B., Shenoy, H.G., Horgan, K. and Hughes, T.A. (2011) Surgery induced immunosuppression. Surgeon, 9, 38-43. http://dx.doi.org/10.1016/j.surge.2010.07.011

[16] Hiraga, A., Mori, M., Ogawara, K., Misawa, S., Kanesaka, T., Koga, M., et al. (2005) Recovery of patterns and long term prognosis for axonal Guillain-Barre syndrome. Journal of Neurology Neurosurgery \& Psychiatry, 76, 719722. http://dx.doi.org/10.1136/jnnp.2004.051136
[17] Tamura, N., Kuwabara, S., Misawa, S., Kanai, K., Nakata, M., Sawai, S., et al. (2007) Time course of axonal regeneration in acute motor axonal neuropathy. Muscle \& Nerve, 35, 793-795. http://dx.doi.org/10.1002/mus.20729

[18] Hughes, R.A. and Cornblath, D.R. (2005) Guillain-Barre syndrome. Lancet, 366, 1653-1666. http://dx.doi.org/10.1016/S0140-6736(05)67665-9

[19] Albers, J.W., Donofrio, P.D. and McGonagle, T.K. (1985) Sequential electrodiagnostic abnormalities in acute inflammatory demyelinating polyradiculoneuropathy. Muscle \& Nerve, 8, 528-539. http://dx.doi.org/10.1002/mus.880080609

[20] Hiraga, A., Kuwabara, S., Ogawara, K., Misawa, S., Kanesaka, T., Koga, M., et al. (2005) Patterns and serial changes in electrodiagnostic abnormalities of axonal Guillain-Barre syndrome. Neurology, 64, 856-860. http://dx.doi.org/10.1212/01.WNL.0000153071.71335.E9

[21] Sakakibara, R., Hattori, T., Kuwabara, S., Yamanishi, T. and Yasuda, K. (1997) Micturitional disturbance in patients with Guillain-Barre syndrome. Journal Neurology Neurosurgery \& Psychiatry, 63, 649-653. http://dx.doi.org/10.1136/jnnp.63.5.649

[22] Sakakibara, R., Uchiyama, T., Tamura, N., Kuwabara, S., Asahina, M. and Hattori, T. (2007) Urinary retention and sympathetic sphincter obstruction in axonal GuillainBarre syndrome. Muscle \& Nerve, 35, 111-115. http://dx.doi.org/10.1002/mus.20639

[23] Sawai, S., Sakakibara, R., Uchiyama, T., Liu, Z., Yamamoto, T., Ito, T., et al. (2007) Acute motor axonal neuropathy presenting with bowel, bladder, and erectile dysfunction. Journal of Neurology, 254, 250-252. http://dx.doi.org/10.1007/s00415-006-0336-3

[24] Parys, B.T., Woolfenden, K.A. and Parsons, K.F. (1990) Bladder function after simple hysterectomy; urodynamic and neurological evaluation. European Urololgy, 17, 129133.

[25] Cheek, W.R., Anchondo, H., Raso, E. and Scott, R. (1973) Neurogenic bladder and the lumbar spine. Urology, 2, 30-33. http://dx.doi.org/10.1016/0090-4295(73)90210-0 